\title{
Is single-dose prophylactic gentamicin associated with acute kidney injury in patients undergoing cardiac surgery? A matched-pair analysis
}

\author{
Dorthe Viemose Nielsen, MD, ${ }^{\mathrm{a}}$ Maria Fedosova, MS, ${ }^{\mathrm{a}}$ Vibeke Hjortdal, MD, PhD, DMSc, ${ }^{\mathrm{b}}$ and \\ Carl-Johan Jakobsen, MD ${ }^{\mathrm{a}}$
}

\begin{abstract}
Objective: Although aminoglycoside treatment has been associated with nephrotoxic effects, single-dose gentamicin has been considered safe in surgery. However, the effect of a single-dose prophylactic aminoglycoside on the risk of acute kidney injury among patients undergoing cardiac surgery remains uncertain.
\end{abstract}

\begin{abstract}
Methods: A population-based cohort study with matched-pair analysis of 2892 consecutive patients undergoing cardiac surgery at Aarhus University Hospital, Denmark, was performed. Two different prophylactic antibiotic regimens were used during the study period. The patients exposed to a single dose of prophylactic gentamicin were compared with those had not received gentamicin. Statistical analysis for matched data was performed. The data were retrieved from the Western Denmark Heart Registry.
\end{abstract}

Results: Matching resulted in 668 patient pairs. Patients receiving gentamicin had higher maximum serum creatinine during the first postoperative 72 hours (median, 96 vs $90 \mathrm{mmol} / \mathrm{L} ; P=.0002$ ). Also, a greater fraction of patients receiving gentamicin developed acute kidney injury according to the Acute Kidney Injury Network criteria $(22 \%$ vs $17 \% ; P=.02)$. The hospital length of stay was shorter in the control group (5.0 vs 5.6 days; $P<.0001)$. No difference was found in the incidence of patients requiring postoperative dialysis, 30 -day and 1 -year mortality, or the incidence of sternal infection between the 2 groups.

Conclusions: A single-dose prophylactic aminoglycoside in adult cardiac surgery patients was associated with an increased risk of acute kidney injury but not with a greater frequency of postoperative dialysis or mortality. No differences in the incidence of sternal infections between groups were observed. (J Thorac Cardiovasc Surg 2014;148:1634-9)

\begin{abstract}
Aminoglycoside treatment in cardiac surgery patients remains controversial. However, in an effort to reduce the postoperative infection rate, aminoglycosides have been used as standard prophylactic antibiotics in some cardiac centers. The nephrotoxic effect of aminoglycosides is a major concern, and aminoglycoside therapy is currently considered one of the most frequent causes of iatrogenic renal failure. ${ }^{1}$ Aminoglycoside toxicity depends more on the duration of the therapeutic levels than on the peak levels. Thus, single-dose prophylactic aminoglycoside has been considered safe, although a few early animal studies have cautiously concluded that even a moderate single dose can be nephrotoxic. ${ }^{2,3}$ Special concern is warranted for patients undergoing cardiac surgery with cardiopulmonary bypass (CPB), because aminoglycoside treatment can potentiate CPB-induced acute kidney injury (AKI). ${ }^{4}$

From the Departments of Anesthesiology and Intensive Care ${ }^{\mathrm{a}}$ and Cardiothoracic and Vascular Surgery, ${ }^{\mathrm{b}}$ Aarhus University Hospital, Aarhus, Denmark.

Disclosures: Authors have nothing to disclose with regard to commercial support.

Received for publication Jan 15, 2014; revisions received May 16, 2014; accepted for publication May 22, 2014; available ahead of print Aug 20, 2014.

Address for reprints: Dorthe Viemose Nielsen, MD, Department of Anesthesiology and Intensive Care, Aarhus University Hospital, Brendstrupgaardsvej 100, Aarhus, 8200 Aarhus N, Denmark (E-mail: dorthe.viemose.nielsen@skejby.rm.dk). $0022-5223 / \$ 36.00$

Copyright (c) 2014 by The American Association for Thoracic Surgery http://dx.doi.org/10.1016/j.jtcvs.2014.05.090
\end{abstract}

Furthermore, aminoglycosides have several interactions with the drugs routinely prescribed for patients with cardiac disease. Diuretics can amplify the risk of nephrotoxity, and long-term use of nonsteroidal anti-inflammatory drugs can reduce renal excretion of the aminoglycoside, potentiating its half-life and narrowing the therapeutic index. Finally, cardiac surgery patients are often elderly, with potentially impaired cellular repair ability and regeneration after exposure to aminoglycosides. ${ }^{5,6}$

No previous studies have investigated the effect of singledose prophylactic aminoglycoside on postoperative AKI among cardiac surgery patients. At our cardiac unit, the preoperative prophylactic antibiotic regimen was changed in November 2009, with the addition of a single dose of $240 \mathrm{mg}$ gentamicin to the previous regimen of dicloxacillin $1 \mathrm{~g} \times 2$ and single-dose teicoplanin $400 \mathrm{mg}$ on the day of surgery. The aim of our study was to examine whether patients receiving a single dose of prophylactic gentamicin had a greater risk of postoperative AKI compared with patients who had not received prophylactic gentamicin.

\section{METHODS}

The Danish Data Protection Agency approved the study. We used population-based healthcare databases, using the civil registry number assigned to each Danish citizen at birth to link the data. ${ }^{7}$ 


\section{Abbreviations and Acronyms \\ AKI = acute kidney injury \\ $\mathrm{CKD}=$ chronic kidney disease \\ $\mathrm{CPB}=$ cardiopulmonary bypass \\ ICU = intensive care unit \\ WDHR $=$ Western Danish Heart Registry}

\section{Study Population}

Computerized data from the Western Danish Heart Registry (WDHR) was used to identify all consecutive patients undergoing cardiac surgery at Aarhus University Hospital from 18 months before the change in the preoperative prophylactic antibiotics to 2 years after. The WDHR is a regional Web-based mandatory registry that includes detailed information on patient history, procedure type, peri- and postoperative management, including inotropic therapy, and in-hospital complications. Data quality is ensured using automatic validation rules at data entry, combined with systematic validation procedures and random spot checks of the data after entry. We identified 3388 procedures during the study period. Double entries $(n=44)$, transcutaneous aortic valve insertion, endocarditis procedures $(\mathrm{n}=335)$, patients with insufficient laboratory values or without a civil registration code $(\mathrm{n}=33)$, and those with a preoperative creatinine $>200 \mu \mathrm{mol} / \mathrm{L}(\mathrm{n}=84)$ were excluded, leaving 2892 patients for analysis.

\section{Anesthesia Protocols and Perioperative Procedures}

According to the hospital guidelines, all preoperative cardiac medications were continued until the morning of surgery, except for angiotensin-converting enzyme inhibitors and platelet inhibitors, including aspirin. The latter were continued in patients with acute coronary syndrome. Anesthesia was primarily administered intravenously, with propofol, sufentanil, and rocuronium. Routine surgical and cardioprotective techniques were used, including crystalloid or cold blood cardioplegia. Most of the patients were maintained with a normothermic or slightly hypothermic temperature. Whether coronary artery bypass surgery was done on- or off-pump was left to the discretion of the attending surgeon. No fixed postoperative treatment regimen was in use concerning either pharmaceutical or mechanical support.

\section{Aminoglycoside Treatment}

Before November 2009, all patients received a single dose of teicoplanin $400 \mathrm{mg}$ intravenously 1 hour before surgery and dicloxacillin $1 \mathrm{~g}$ intravenously at the induction of anesthesia and repeated 8 hours later. After November 2009, this regimen was supplemented with gentamicin $240 \mathrm{mg}$ intravenously (those weighing $>120 \mathrm{~kg}$ received $480 \mathrm{mg}$ ) at the induction of anesthesia.

Information about aminoglycoside treatment was obtained from the departmental electronic patient management system.

\section{Patient and Procedure Characteristics}

The patients and procedures were characterized using the EuroSCORE I variables, including ${ }^{8}$ age, sex, and grouped EuroSCORE variables. The variables included patient-related factors (chronic pulmonary disease, extracardiac arteriopathy, neurologic dysfunction disease, previous cardiac surgery, serum creatinine, active endocarditis, critical preoperative state), cardiac-related factors (unstable angina, recent myocardial infarction, pulmonary hypertension, left ventricular function), procedure-related factors (emergency surgery, surgery other than coronary artery bypass grafting, aortic surgery, and ventricular septal defect surgery), and insulin-dependent diabetes, perioperative use of aprotinin, and extracorporeal circulation time (off pump vs $<120$ minutes vs $\geq 120$ minutes).

\section{Patient Outcomes}

Serum creatinine was routinely measured within the first 6 hours postoperatively after arrival at the intensive care unit (ICU) and on the subsequent postoperative days until discharge. The serum creatinine values were obtained from the hospital laboratory information database.

The patient outcomes were the absolute and relative changes in postoperative serum creatinine $\leq 10$ days after surgery and the postoperative development of stage 1 AKI using the Acute Kidney Injury Network ${ }^{9}$ criteria: an increase in serum creatinine of $>0.3 \mathrm{mg} / \mathrm{dL}(>26.5 \mu \mathrm{mol} / \mathrm{L})$ or an increase of $50 \%$ to $200 \%$ from baseline. However, owing to the hemodilution normally found in connection with $\mathrm{CPB}$, the window for evaluation of serum creatinine was increased to 72 hours after surgery, as reported previously. ${ }^{10}$ The secondary outcome measures were temporary or permanent in-hospital dialysis, ICU stay, in-hospital stay, 30-day and 1-year mortality, and sternal infections.

Incident dialysis was registered in the WDHR by surgeons before discharge to the local hospital. The ICU and hospital length of stay were also registered in the WDHR. The date of death was obtained through the Civil Registration System, where the exact date of death for each citizen is registered. All patients in the study cohort were followed up for $\geq 1$ year. Sternal infections were diagnosed as deep infection and verified by the bacteriologic findings and were registered by the surgeons in the WDHR before discharge.

\section{Statistical Analysis}

Because the patient characteristics were not comparable for all parameters across the 2 study periods, we used individual matching to identify 1 control patient who had not received gentamicin as prophylaxis for each patient who had received gentamicin as single-dose prophylaxis. Exact matching was performed for the following primary factors potentially related to the risk of postoperative AKI: EuroSCORE criteria (age score [0-7], sex [0-1], patient factors [0-5], cardiac factors [0-7], procedure factors [0-7]); overall EuroSCORE (0-17); perioperative aprotinin (yes vs no); and extracorporeal circulation time (off pump $=0$; $<120$ minutes $=1 ;$ and $\geq 120$ minutes $=2$ ). The secondary factors were also matched: insulin-dependent diabetes (yes vs no) and surgery type ( 6 types: coronary artery bypass grafting, aortic valve replacement, mitral valve replacement, single other, complex, aortic). In the case of $>1$ possible match using the primary matching criteria, the patients with an exact match for diabetes and surgery type were selected (516 pairs) or those with a match providing an equal number of the 2 secondary criteria in each overall EuroSCORE group (152 pairs). An eventually tertiary criterion was the closest age in years. The matching process resulted in 668 patients in each group (Table 1).

Wilcoxon's signed rank test was used to compare the gentamicin-treated patients and their matched controls for changes in serum creatinine, ICU stay, and hospital length of stay. McNemar's test and conditional logistic regression analysis were used to compare the gentamicin-treated patients and their matched controls for the occurrence of AKI, temporary or permanent in-hospital dialysis, 30-day and 1-year mortality, sternal infections, and risk factors for the development of AKI. To further reduce the risk of confounding owing to preoperative renal status, the preoperative creatinine and preoperative medication with angiotensin-convertingenzyme inhibitors were included in the regression analysis. Analyses were performed using MedCalc software, version 12.3 (Mariakerke, Belgium). Conditional regression analysis was performed using Stata IC 10 (StataCorp, College Station, Tex).

\section{RESULTS}

The demographic data and perioperative risk factors related to aminoglycoside treatment are listed in Table 1. The characteristics are presented as independent variables 
TABLE 1. Characteristics of overall consecutive cohort and matched cohort

\begin{tabular}{|c|c|c|c|c|c|c|}
\hline \multirow[b]{2}{*}{ Characteristic } & \multicolumn{3}{|c|}{ Primary consecutive cohort } & \multicolumn{3}{|c|}{ Matched cohort } \\
\hline & Control & Gentamicin & $P$ value & Control & Gentamicin & $P$ value \\
\hline Patients (n) & 1269 & 1623 & - & 668 & 668 & - \\
\hline Age (y) & $64.8 \pm 13.4$ & $65.8 \pm 13.6$ & $.0510^{*}$ & $64.0 \pm 13.8$ & $64.1 \pm 13.8$ & $.8925 \dagger$ \\
\hline Female sex & $377(29.7)$ & $489(30.1)$ & $.8381 \ddagger$ & $185(27.7)$ & $185(27.7)$ & $.9505 \S$ \\
\hline Age and sex score & $2.38 \pm 1.87$ & $2.54 \pm 1.91$ & $.0249^{*}$ & $2.28 \pm 1.88$ & $2.28 \pm 1.88$ & $1.0^{\dagger}$ \\
\hline Patient factors & $0.79 \pm 1.35$ & $0.83 \pm 1.43$ & $.4429 *$ & $0.39 \pm 0.91$ & $0.39 \pm 0.91$ & $1.0^{\dagger}$ \\
\hline Cardiac factors & $1.09 \pm 1.65$ & $1.26 \pm 1.75$ & $.0075^{*}$ & $0.72 \pm 1.32$ & $0.72 \pm 1.32$ & $1.0^{\dagger}$ \\
\hline Off-pump & $81(6.4)$ & $184(11.3)$ & - & $38(5.7)$ & $38(5.7)$ & - \\
\hline $\mathrm{ECC}<120 \mathrm{~min}$ & $924(72.8)$ & $1014(62.5)$ & $<.0001 \ddagger$ & $524(78.4)$ & $524(78.4)$ & $1.0 \ddagger$ \\
\hline $\mathrm{ECC} \geq 120 \mathrm{~min}$ & $264(20.8)$ & $424(26.1)$ & - & $106(15.9)$ & $106(15.9)$ & - \\
\hline Procedure factors & $1.36 \pm 1.52$ & $1.42 \pm 1.69$ & $.3919 *$ & $1.13 \pm 1.32$ & $1.13 \pm 1.32$ & $1.0 \dagger$ \\
\hline EuroSCORE & $5.63 \pm 3.50$ & $6.05 \pm 3.72$ & $.0019^{*}$ & $4.52 \pm 2.78$ & $4.52 \pm 2.78$ & $1.0 \dagger$ \\
\hline Aprotinin & $93(7.3)$ & $151(9.3)$ & $.0667 \ddagger$ & $31(4.6)$ & $31(4.6)$ & $.8973 \S$ \\
\hline Insulin-dependent DM & $61(4.8)$ & $85(5.2)$ & $.6580 \ddagger$ & $20(3.0)$ & $20(3.0)$ & $.8638 \S$ \\
\hline Preoperative creatinine $(\mu \mathrm{mol} / \mathrm{L})$ & $84 \pm 25$ & $87 \pm 23$ & $.0019^{*}$ & $82 \pm 22$ & $84 \pm 21$ & $.1075 \dagger$ \\
\hline CABG & $572(45.1)$ & $724(44.6)$ & - & $332(49.7)$ & $321(48.1)$ & - \\
\hline AVR & $242(19.1)$ & $318(19.6)$ & - & $159(23.8)$ & $166(24.9)$ & - \\
\hline CABG plus AVR & $120(9.5)$ & $146(9.0)$ & - & $53(7.9)$ & $51(7.6)$ & - \\
\hline MVR & $68(5.4)$ & $85(5.2)$ & $.8066 \ddagger$ & $29(4.3)$ & $32(4.8)$ & .9949 \\
\hline Single other procedure & $118(9.3)$ & $137(8.4)$ & - & $55(8.2)$ & $58(8.7)$ & - \\
\hline Complex procedure & $78(6.1)$ & $123(7.6)$ & - & $20(3.0)$ & $19(2.8)$ & - \\
\hline Aortic surgery & $71(5.6)$ & $90(5.5)$ & - & $20(3.0)$ & $22(3.3)$ & - \\
\hline
\end{tabular}

Data presented as n, mean \pm standard deviation, or n (\%). ECC, Extracorporeal circulation; $D M$, diabetes mellitus; $C A B G$, coronary artery bypass grafting; $A V R$, aortic valve replacement; MVR, mitral valve repair or replacement; EuroSCORE, European System for Cardiac Operative Risk Evaluation. *Independent samples $t$ test. $\dagger$ Paired samples $t$ test. $\ddagger$ Chi-square test. $\S \mathrm{McNemar}$ test.

and, where relevant, were combined into computed score indices. After matching, 668 patients were in each group, and the patients were comparable for all evaluated parameters (Table 1). In the matched cohort of 1336 patients, $255(19 \%)$ developed AKI within the first 72 hours postoperatively. The patient outcomes stratified by gentamicin treatment are listed in Table 2.

The patients receiving gentamicin had greater relative changes in serum creatinine during the first 72 postoperative hours (Figure 1), although no difference was found in the relative changes after 72 hours. The calculated creatinine clearance (mL/min [Cockcroft-Gault formula]) showed significantly lower minimal values postoperatively in the gentamicin group (median, 80; interquartile range, 76-83, vs median, 85 ; interquartile range, $81-88 ; P=.0001$, Wilcoxon's signed rank test). No difference was found in the incidence of new-onset dialysis between the 2 groups. The in-hospital stay was slightly longer for the gentamicin group. The incidence of sternal infections was the same in the 2 groups (control group, $1.2 \%$, vs gentamicin group, $0.8 ; P=.5811)$. We did not find a significant difference in 1-year mortality, because the study was not powered to include mortality as the primary outcome.

To avoid a bias resulting from preoperative renal dysfunction, all patients with a serum creatinine $>200$ $\mu \mathrm{mol} / \mathrm{L}$ were excluded before the matching process. Despite this, some patients had an impaired calculated clearance preoperatively and were found to be more prone to AKI if exposed to gentamicin (Table 3). The difference between the treatment groups was not significant in patients with a clearance of $<50 \mathrm{~mL} / \mathrm{min}$, probably owing to the very few patients in this category.

We could not demonstrate differences in AKI between groups for the patients weighing $<60 \mathrm{~kg}$ (single dose $>4 \mathrm{mg} / \mathrm{kg}$ ) and those weighing $>60 \mathrm{~kg}$ (AKI frequency,

TABLE 2. Single-dose prophylactic gentamicin and patient outcomes

\begin{tabular}{|c|c|c|c|}
\hline Patient outcome & $\begin{array}{c}\text { Control } \\
(n=668)\end{array}$ & $\begin{array}{c}\text { Gentamicin } \\
(n=668)\end{array}$ & $\begin{array}{c}P \\
\text { value }\end{array}$ \\
\hline Development of AKI & $110(16.5)$ & $145(21.7)$ & $.0240^{*}$ \\
\hline Maximal serum creatinine & & & $.0002 \dagger$ \\
\hline Median & 90 & 96 & \\
\hline IQR & $76-108$ & $81-122$ & \\
\hline New dialysis & $12(1.8)$ & $12(1.8)$ & $1.0^{*}$ \\
\hline Hospital LOS (d) & & & $<.0001 \dagger$ \\
\hline Median & 5.0 & 5.6 & \\
\hline IQR & $4.0-6.8$ & $4.9-7.4$ & \\
\hline ICU LOS (h) & & & $.3225 \dagger$ \\
\hline Median & 21.4 & 21.3 & \\
\hline IQR & $19.2-22.8$ & $18.8-22.8$ & \\
\hline 30-d mortality & $8(1.2)$ & $9(1.3)$ & $1.0^{*}$ \\
\hline 1-y mortality & $16(2.4)$ & $17(2.5)$ & $1.0^{*}$ \\
\hline Sternal infection & $8(1.2)$ & $5(0.8)$ & $.5811^{*}$ \\
\hline
\end{tabular}




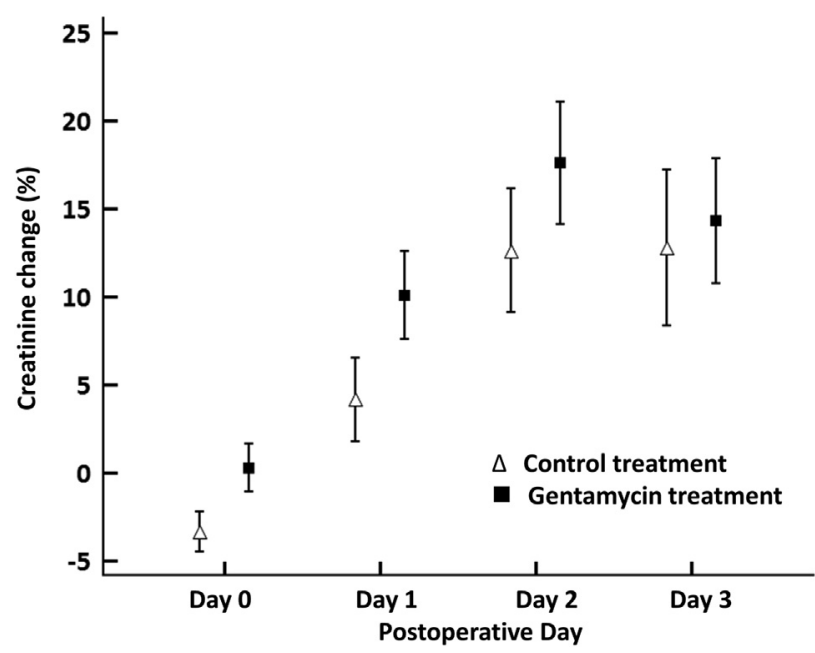

FIGURE 1. Relative changes in serum creatinine from day of surgery according to treatment groups. Day 0, first postoperative creatinine measurement $(P=.017$, repeated measures analysis of variance).

$18.1 \%$ vs $19.4 \% ; P=.8305$ ). However, we could demonstrate a correlation between patient weight and the lowest creatinine clearance within the first 72 postoperative hours $(\mathrm{r}=0.435 ; P<.0001)$, indicating that the decrease in postoperative renal function was dose dependent.

Conditional logistic regression showed that preoperative serum creatinine $>120 \mu \mathrm{mol} / \mathrm{L}$ and single-dose prophylactic gentamicin were independently associated with an increased risk of postoperative AKI (Table 4).

We could not demonstrate an overall greater frequency of AKI after on-pump surgery versus off-pump surgery. However, among the on-pump patients, AKI occurred more frequently in the gentamicin group (21\% vs $17 \%$; $P=.0387$; McNemar test).

\section{DISCUSSION}

Our findings have indicated that single-dose prophylactic gentamicin is associated with an increased risk of the development of stage 1 AKI. Patients with decreased preoperative creatinine clearance were more vulnerable to the nephrotoxic effect of prophylactic gentamicin; however, the data were not granular enough to determine the level of decreased preoperative creatinine clearance to warrant use

TABLE 3. Development of AKI according to preoperative creatinine clearance* and gentamicin treatment

\begin{tabular}{lccr}
\hline $\begin{array}{l}\text { Preoperative creatinine } \\
\text { clearance (mL/min) }\end{array}$ & Control (\%) & Gentamicin $(\%)$ & $\boldsymbol{P}$ value \\
\hline$>85$ & 11.5 & 14.7 & $<.0001 \dagger$ \\
$50-85$ & 20.7 & 28.3 & $<.0001 \dagger$ \\
$<50$ & 40.0 & 48.9 & $.5322 \dagger$ \\
$P$ value & $<.0001 \ddagger$ & $<.0001 \ddagger$ & \\
\hline
\end{tabular}

*Cockcroft-Gault calculation formula. $\dagger$ McNemar test. $\ddagger$ Chi-square test.
TABLE 4. Adjusted risk of acute kidney injury

\begin{tabular}{lr}
\hline \multicolumn{1}{c}{ Risk factor } & OR $(\mathbf{9 5} \% \mathbf{C I}) *$ \\
\hline Single-dose prophylactic gentamicin & $1.38(1.02-1.87)$ \\
Preoperative creatinine $>120 \mu \mathrm{mol} / \mathrm{L}$ & $3.5(1.50-8.37)$ \\
Preoperative medication with ACEI & $1.14(0.71-1.82)$ \\
\hline$O R$, Odds ratio; $C I$, confidence interval; $A C E I$, angiotensin-converting enzyme inhib- \\
itor. *Conditional regression analysis.
\end{tabular}

of prophylactic gentamicin. The frequency of postoperative dialysis was the same in the 2 groups and was in accordance with previous reports of the incidence of postoperative dialysis after cardiac surgery. ${ }^{11-13}$ Prophylactic gentamicin did not seem to have any effect on the development of sternal infection, because the incidence was similar in the 2 groups. However, the hospital length of stay was slightly longer in the gentamicin group.

We have previously reported perioperative aminoglycoside treatment to be associated with a greater risk of postoperative dialysis ${ }^{10}$; however, in that study, the patients had been treated with $>1$ gentamicin dose, and gentamicin was not standard antibiotic treatment, making it difficult to draw conclusions regarding the possible nephrotoxic effect of single-dose prophylactic gentamicin in cardiac surgery patients.

Few other studies have been considering the effect of single-dose gentamicin in surgical patients. Craig and colleagues ${ }^{14}$ found no difference in creatinine levels between the study and control groups in patients with a fractured femoral neck. Sparse knowledge of their patients' weight prevented the investigators from calculating the creatinine clearance. ${ }^{14}$ Furthermore, control matching by comorbidity was restricted to sex and age variables. Solgaard and colleagues ${ }^{15}$ observed a transient reduction in renal function in patients undergoing femoral neck surgery who received $2 \mathrm{~g}$ dicloxacillin and $240 \mathrm{mg}$ gentamicin. Because dicloxacillin has been associated with nephrotoxicity, it was difficult to draw a firm conclusion. ${ }^{15}$ Among patients undergoing cardiac surgery, aminoglycoside treatment has been evaluated with simultaneous administration of other potentially nephrotoxic drugs, with the combinations associated with an increase in renal failure. ${ }^{16,17}$

$\mathrm{CPB}$ and the subsequent reestablishment of pulsatile flow can result in an ischemic-reperfusion injury in the renovascular bed. This could be one of the main reasons for AKI after cardiac surgery, and it has been shown in rats that aminoglycoside exacerbates postischemic AKI. ${ }^{4}$ Other studies have suggested that perioperative aminoglycosides in cardiac surgery are associated with changes in elimination kinetics, followed by an increase in serum creatinine. ${ }^{18,19}$ Lewis and colleagues ${ }^{19}$ demonstrated a significant correlation between the elimination time and length of CPB. They concluded that gentamicin excretion was delayed after $\mathrm{CPB}$ and that a dose $>4 \mathrm{mg} / \mathrm{kg}$ increased 
the risk of nephrotoxicity. ${ }^{19}$ The correlation between weight and lowest creatinine clearance in the present study may support these findings.

In the present study, no difference was found in the overall rate of AKI between the on- and off-pump procedures; however, more patients in the gentamicin group developed AKI after on-pump surgery than did on-pump patients who had not received gentamicin, supporting the hypothesis of previous studies. Although the present changes in creatinine among patients receiving gentamicin were only transient, and no difference could be measured regarding in-hospital dialysis, recent studies have indicated that even minimal increases in serum creatinine values after cardiac surgery are associated with long-term adverse outcomes, including mortality, progression of chronic kidney disease (CKD), and incident CKD. ${ }^{20-22}$ Ishani and colleagues $^{20}$ showed that patients with a peak creatinine level of $1 \%$ to $24 \%$ increase from baseline (comparable to the present increase among gentamicin-treated patients) had an increased risk of CKD development (hazards ratio, 2.1), progression of CKD stage (hazard ratio, 2.5), and mortality (hazard ratio, 1.4) at 3 months postoperatively compared with a reference group with no increase in creatinine. $^{20}$

Several patient- and procedure-related factors are known to be predictors of postoperative renal failure: age, extracorporeal circulation time, EuroSCORE, and cardiac variables. ${ }^{10-13}$ The role of aprotinin on postoperative renal failure remains a matter of debate. ${ }^{23-25}$ Because we noted an increase in the use of aprotinin during the study period, aprotinin was included in the matching process with the previously mentioned risk factors. The matching of controls to those exposed to gentamicin using these most common risk factors for postoperative AKI was intended to reduced the risk of residual confounding. Preoperative renal failure is a well-known predictor of postoperative mortality and renal failure morbidity. ${ }^{26,27}$ Ideally, matching using preoperative clearance would have been preferred; however, patient weight was not registered for all patients in the database. Thus, the preoperative serum creatinine was included in the regression model.

We believe we created 2 analytical cohorts (gentamicin vs no gentamicin) that were very similar in measurable patient, procedure, and preoperative renal characteristics. Despite these similar characteristics, patients exposed to gentamicin were more prone to develop AKI stage 1 after cardiac surgery.

\section{Study Strengths and Limitations}

The main strength of our study was its prospective population-based design with complete follow-up data for clinical outcomes and, consequently, a low risk of selection and information bias. Furthermore, detailed and complete data for the patient- and procedure-related characteristics were available, and matching created fully comparable groups. However, ours was a historical observation study; thus, the conclusions should be interpreted with caution. Although the matching adjusted for a wide range of possible confounding factors in the data analysis, the matching also excluded the sickest patients in both groups, and we could not entirely exclude the possibility that residual or unaccounted confounding might have influenced the study findings. The hospital length of stay in the present study refers to the stay at our institution. Because a high number of patients are normally referred to their home hospital after 4 to 5 days, the actual length of hospitalization was longer, and we could not exclude that changes in policies or logistics could have occurred during the observation time.

Our results showed that the consensus criteria for AKI are difficult to apply to cardiac surgery patients, even after we extended the time window from 48 to 72 hours, because relatively many patients had higher serum creatinine after 72 hours postoperatively.

\section{CONCLUSIONS}

In the present cohort study of 668 matched pairs of adult cardiac surgery patients, a single dose of prophylactic aminoglycoside was associated with a greater risk of AKI and longer hospitalization but had no effect on postoperative dialysis, mortality, or incidence of sternal infection. Patients with preoperative impaired renal function were more sensitive to the nephrotoxic effects of gentamicin.

\section{References}

1. Davidman M, Olson P, Kohen J, Leither T, Kjellstrand C. Iatrogenic renal disease. Arch Intern Med. 1991;151:1809-12.

2. Pahl MV, Vaziri ND, Leitman PS, Nguyen T. Effect of renal mass reduction on kidney tissue concentration of gentamicin following a single dose. Res Commun Chem Pathol Pharmacol. 1988;60:405-8.

3. Riviere JE, Hinsman EJ, Coppoc GL, Carlton WW. Single dose gentamicin nephrotoxicity in the dog: early functional and ultrastructural changes. Res Commun Chem Pathol Pharmacol. 1981;33:403-18.

4. Zager RA. Gentamicin effects on renal ischemia/reperfusion injury. Circ Res. 1992;70:20-8.

5. Raveh D, Kopyt M, Hite Y, Rudensky B, Sonnenblick M, Yinnon AM. Risk factors for nephrotoxicity in elderly patients receiving once-daily amino-glycosides. QJM. 2002;95:291-7.

6. Cherry KE, Morton MR. Drug sensitivity in older adults: the role of physiologic and pharmacokinetic factors. Int J Aging Hum Dev. 1989;28:159-74.

7. Pedersen CB, Gotzsche H, Moller JO, Mortensen PB. The Danish Civil Registration System: a cohort of eight million persons. Dan Med Bull. 2006;53:441-9.

8. Roques F, Nashef SAM, Michel P, Gauducheau E, de Vincentiis C, Baudet E, et al. Risk factors and outcome in European cardiac surgery: analysis of the EuroSCORE multinational database of 19,030 patients. Eur J Cardiothorac Surg. 1999; 15:816-23.

9. Mehta RL, Kellum JA, Shah SV, Molitoris BA, Ronco C, Warnock DG, et al; Acute Kidney Injury Network. Acute Kidney Injury Network: report of an initiative to improve outcomes in acute kidney injury. Crit Care. 2007;11:R31.

10. Nielsen DV, Hjortdal V, Johnsen SP, Jakobsen C-J. Perioperative aminoglycoside treatment is associated with higher incidence of postoperative dialysis in adult cardiac surgery patients. J Thorac Cardiovasc Surg. 2011;142:656-61.

11. Filsoufi F, Rahmanian PB, Castillo JG, Silvay G, Carpentier A, Adams DH. Predictors and early and late outcomes of dialysis-dependent patients in contemporary cardiac surgery. J Cardiothorac Vasc Anesth. 2008;22:522-9. 
12. Mehta RH, Grab JD, O’Brien SM, Bridges CR, Gammie JS, Haan CK, et al; Society of Thoracic Surgeons National Cardiac Surgery Database Investigators. Bedside tool for predicting the risk of postoperative dialysis in patients undergoing cardiac surgery. Circulation. 2006;114:2208-16.

13. Bahar I, Akgul A, Ozatik MA, Vural KM, Demirbag AE, Boran M, et al. Acute renal failure following open heart surgery: risk factors and prognosis. Perfusion. 2005;20:317-22.

14. Craig P, Starks I, Bancroft G, Roberts P. Is prophylactic gentamicin associated with acute kidney injury in patients undergoing surgery for fractured neck of femur? Injury. 2012;43:2152-5.

15. Solgaard L, Tuxoe JI, Mafi M, Due Olsen S, Toftegaard Jensen T. Nephrotoxicity by dicloxacillin and gentamicin in 163 patients with intertrochanteric hip fracture. Int Orthop. 2000;24:155-7.

16. Mercieri M, Mercieri A, Tritapepe L, Ruggeri M, Arcioni R, Repetto M, et al. High-dose aprotinin with gentamicin-vancomycin antibiotic prophylaxis increases blood concentrations of creatinine and cystatin $\mathrm{C}$ in patients undergoing coronary artery bypass grafting. Br J Anaesth. 1999;82:531-6.

17. Schneider M, Valentine S, Clarke GM, Newman MAJ, Peacock J. Acute renal failure in cardiac surgical patients, potentiated by gentamicin and calcium. Anaesth Intensive Care. 1996;24:647-50.

18. Klamerus KJ, Rodvold KA, Silverman NA, Levitsky S. Effect of cardiopulmonary bypass on vancomycin and netilmicin disposition. Antimicrob Agents Chemother. 1988;32:631-5.

19. Lewis DR, Longman RJ, Wisheart JD, Spencer RC, Brown NM. The pharmacokinetics of a single dose of gentamicin $(4 \mathrm{mg} / \mathrm{kg}$ ) as prophylaxis in cardiac surgery requiring cardiopulmonary bypass. Cardiovasc Surg. 1999;7:398-401.
20. Ishani A, Nelson D, Clothier B, Schult T, Nugent S, Greer N, et al. The magnitude of acute serum creatinine increase after cardiac surgery and the risk of chronic kidney disease, progression of kidney disease, and death. Arch Intern Med. 2011;171:226-33.

21. Kolli H, Rajagopalam S, Patel N, Ranjan R, Venuto R, Lohr J, et al. Mild acute kidney injury is associated with increased mortality after cardiac surgery in patients with eGFR $<60 \mathrm{~mL} / \mathrm{min} / 1.73 \mathrm{~m}(2)$. Ren Fail. 2010;32: 1066-72.

22. Tolpin DA, Collard CD, Lee V, Virani SS, Allison PM, Elayda MA, et al Subclinical changes in serum creatinine and mortality after coronary artery bypass grafting. J Thorac Cardiovasc Surg. 2012;143:682-8.

23. Mangano DT, Tudor IC, Dietzel C, Multicenter Study of Perioperative Ischemia Research Group, Ischemia Research and Education Foundation. The risk associated with aprotinin in cardiac surgery. N Engl J Med. 2006;354:353-65.

24. Jakobsen CJ, Sondergaard F, Hjortdal VE, Johnsen SP. Use of aprotinin in cardiac surgery: effectiveness and safety in a population-based study. Eur J Cardiothorac Surg. 2009;36:863-8

25. Howell N, Senanayake E, Freemantle N, Pagano D. Putting the record straight on aprotinin as safe and effective: results from a mixed treatment meta-analysis of trials of aprotinin. J Thorac Cardiovasc Surg. 2013;145:234-40.

26. Miceli A, Bruno VD, Capoun R, Romeo R, Angelini GD, Caputo M. Occult renal dysfunction: a mortality and morbidity risk factor in coronary artery bypass grafting surgery. J Thorac Cardiovasc Surg. 2011;141:771-6.

27. Dhanani J, Mullany DV, Fraser JF. Effect of preoperative renal function on long-term survival after cardiac surgery. J Thorac Cardiovasc Surg. 2013; 146:90-5. 